\title{
Historein
}

Vol $2(2000)$

Heterodoxies: Constructions of Identities and Otherness in Medieval and Early Modern Europe

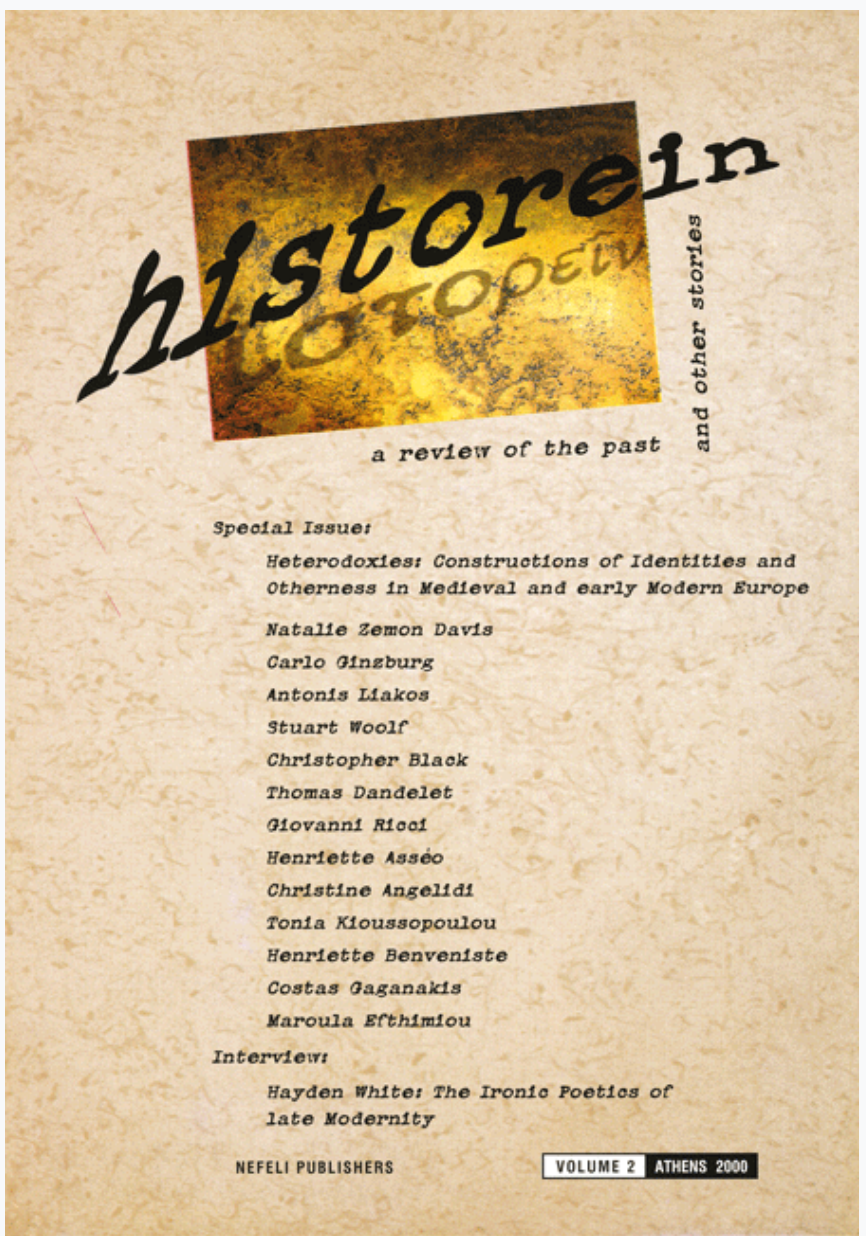

\section{Pratiques de représentation et constructions d'identité: juifs et chrétiens au XIle siècle}

Henriette Benveniste

doi: $10.12681 /$ historein.119

Copyright @ 2012, Henriette Benveniste

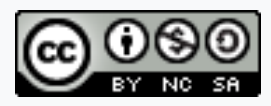

This work is licensed under a Creative Commons Attribution-NonCommercialShareAlike 4.0.

\section{To cite this article:}

Benveniste, H. (2001). Pratiques de représentation et constructions d'identité: juifs et chrétiens au XIle siècle. Historein, 2, 143-156. https://doi.org/10.12681/historein.119 


\section{Pratiques de}

\section{représentation}

\section{et constructions}

\section{d'identité:}

\section{juifs}

et chrétiens

\section{au XIIe siècle}

La question que je me pose dans la présente communication est la suivante: Quel est le regard que les juifs portent sur eux-mêmes et sur les autres selon qu'ils sont persécutés ou vivent en temps de paix? En d'autres termes, quels sont les limites de l'hétérodoxie au XIIe siècle dans le cas des rapports entre juifs et chrétiens? Je me propose d'examiner les traits communs ainsi que les divergences de deux types de récit, des morceaux d'une mosaïque qu'on pourrait appeller "civilisation juive" du XIle siècle, produits dans des conditions différentes qui ont forgé des aspects d'un processus d'identification. ${ }^{1}$

* Le premier type de ces recits expose les massacres de la première croisade, prêchée en 1095, qui a été étroitement liée à une persécution de grande ampleur à l'encontre des juifs. Sur leur route pour Jérusalem, les armées régulières des croisés s'attaquèrent aux juifs le long de la vallée du Rhin en leur imposant le choix suivant: le baptême ou la mort. Nous possédons trois textes, connus sous le nom de "chroniques hébraïques," contemporains des événements ou redigés quelques décénies plus tard, qui présentent les faits avec le souci manifeste de les décrire au plus près. ${ }^{2}$ Le thème central de ces récits est bien l'exaltation du comportement de ces juifs qui, décidés à s'opposer au baptême forcé, firent le choix de se suicider collectivement ou individuellement. De quelle manière le martyre et sa narration ontils pu prendre sens et valeur, à quel ordre de savoir et de symboles se sont-ils référés?

* Les voyages de deux juifs du Xlle siècle forment le deuxième type de récit. Dans son voyage qui dura environ quatorze ans (11591173) Benjamin de Tudèle partit d'Espagne, ${ }^{3}$ 
traversa la France, I'Italie et la Grèce, arriva à Constantinople et de là il se dirigea vers la Mésopotamie et la Palestine; il passa ensuite en Afrique et de l' Égypte il rentra enfin en Espagne. Le second voyageur, Petahia de Ratisbonne ${ }^{4}$ partit de Prague et de 1174 à 1185 il traversa la Pologne et la Russie, il parvint en Crimée et en Arménie, il visita ensuite l'Irak, la Syrie, la Palestine et la Grèce. Benjamin et Petachia partagent une communauté textuelle, mais leurs écritures obéïssent à des traditions différentes qui conditionnent leurs manières de s'approprier les réalités de leurs voyages.

Je me propose alors, plus précisement, d' étudier les récits de persécution ou de voyage sous le prisme (i) des pratiques de mémorisation et de commémoration, (ii) des pratiques d'identification d'un "soi collectif" et (iii) les pratiques de représentation des non juifs et plus particulièrement des chrétiens.

\section{i. Pratiques de mémorisation et de commémoration}

Mémoires et identités se construisent et se transforment dans le temps. Au cœur de toute identité collective il existe un système de reconnaissance de soi et des autres qui s'appuie sur des pratiques de mémorisation et de commémoration; inversement, les choix de la mémoire sont déterminés par cette identité en construction.

Les travaux récents ont montré que les massacres effectués par les croisés n'ont pas entraîné une détérioration du statut des juifs survivants et n'ont pas été le point de départ d'un enchaînement de persécutions. ${ }^{5}$ Cependant, la croisade impose un dilemme violemment nouveau: conversion de masse ou bien massacre collectif. Nous pouvons donc considerer cette persécution comme un tournant au sens où elle a constitué une rupture dans les relations de tolérance qui pouvaient prévaloir dans certains cas. Mais surtout, son souvenir a exercé une influence considérable sur la mémoire juive. Comment le récit traite-il le sujet de la mémoire?

Le fait de raconter un événement est un témoignage pour l'histoire mais aussi un acte de responsabilité à l'égard des martyrs sacrifiés. ${ }^{6}$ II obéit à un genre littéraire mixte constitué à la fois

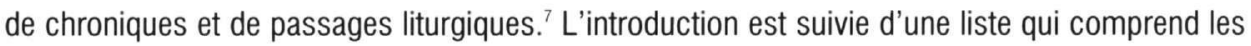
noms des martyrs et la formulation d'un vœu: “Que Dieu se souvienne d'eux pour le bien!” Ceci renvoie à la liturgie de la prière pour les morts, au Kaddish: les vivants doivent se souvenir des morts pour que Dieu s'en souvienne aussi. La remémoration de leurs noms donne un sens à leur sacrifice et renforce la cohésion de la communauté des survivants.

La narration est aussi un acte de piété qui s'adresse à Dieu comme prière et aux juifs comme ordre qui les engage à faire preuve de foi: survivre en tant que juifs pour se souvenir des martyrs. Le sacrifice offert à Dieu scèle une conduite morale où la mort n'est pas une fin. Les martyrs

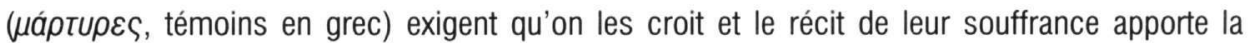
preuve de l'existence de Celui qui donne la vie et la prend. La narration de la catastrophe constitue un exemplum d'autodestruction, lorsque l' autre terme de l' alternative est écartée.

Dans les Memorbücher, les livres du souvenir - ces "cimetières textuels" comme on les désigne 
souvent-les plus vieilles inscriptions datent de 1096. Bien que le but initial de ces récits semble être la légitimation des rituels de mémorisation, ils n'ont pas pu être intégrés aux narrations historiques lues à ra synagogue - peut-être parce qu' ils décrivaient des personnages et des héros concrets. ${ }^{8}$ On a retenu le principe de donner la longue liste des noms des martyrs. Par ailleurs, les récits abondent en citations bibliques qui se nouent de manière indissoluble à l'histoire racontée. Les événements contemporains sont reliés à des histoires bibliques et le fait contemporain est en même temps un fait diachronique. Dans le judaïsme, les faits n'ont ni un statut autonome, ni une valeur de preuve; leur représentation est exemplaire, cependant, les récits de persécution ne se limitent pas aux archétypes empruntés aux histoires bibliques; ${ }^{9}$ dans notre exemple, la citation des noms des martyrs renouvelle la tradition.

La mémorisation est aussi assurée par la forme ritualisée du martyre. Les textes se refèrent à l'image biblique de l'Akedah (Gen.22,2 et suiv); le sacrifice d'Isaac est la référence principale et le prisme à travers lequel l'expérience est vécue. La métaphore, explicite dans le texte, donne lieu à une performance rituelle qui efface le temps aussi bien pour les martyrs que pour les narrateurs.

Par ailleurs, la signification du martyre s'accroche au rêve du Temple qui attirait toujours l'imagination des juifs en Diaspora; ils le vénéraient et vivaient dans l'espoir qu'un jour il serait reconstruit. Le sacrifice dans le Temple, tel qu'il était pratiqué à l'époque biblique est resté l'idéal d'un service de Dieu direct et authentique. Dans l'Ashkenaz médiévale existait une tendance à ritualiser les métaphores textuelles. ${ }^{10}$ En 1096 se développa une idéologie qui tendit à rendre le martyre équivalent au sacrifice des animaux dans le Temple. En attendant les croisés les chefs de famille exercent une fonction de prêtres mais eux-mêmes et leurs familles se présentent en offrandes à Dieu. La spiritualité du Xle siècle, qui a de nombreuses caractéristiques communes chez les juifs et les chrétiens d'Europe, a peut-être facilité la prise de décision, mais celle-ci devait aussi s'appuyer sur une tradition biblique et talmudique solide et familière. Selon la Halacha, la jurisprudence rabbinique, en 1096, les juifs avaient le droit de se tuer pour éviter le baptême. Les exemples ne manquaient pas parmi les personnages héroïques de la tradition biblique. Les récits font des références explicites aux exemples bibliques et même à un commentaire didactique, le Midrash des "dix martyrs." Cependant, en 1096, Dieu n'a pas choisi d'éprouver des individus mais la communauté pieuse toute entière. ${ }^{11}$

Tentons à présent à suivre la perception que les croisés ont eu de l'attitude extreme des juifs, de l'horreur du spectacle des parents tuant leurs enfants? Quiel sens ont-ils donné à ce comportement tous ceux qui en tant que chrétiens étaient habitués à une forme iconographique et littéraire précise de la scène du suicide et à sa figuration la plus répandue: celle où le Désespoir, le vice qui a conduit au suicide, est représenté par Judas pendu à un arbre. Quelles idées sont venues à l'esprit de ces chrétiens qui avaient connaissance de cette littérature où la croisade, qui est aussi une pratique de pénitence, se présentait comme un substitut du suicide et un remède à sa tentation? ${ }^{12}$ Dans quelle mesure le suicide des martyrs pouvait-il correspondre au sacrifice de Jésus? 
L'acte du martyre a une signification politique: au niveau symbolique, il remet en ordre les relations de pouvoir. II constitue une sorte de vengeance purificatrice contre un ennemi qui est surpris par le sacrifice rituel; se rétablit ainsi une supériorité de la victime offensée; les survivants se réunissent autour d'un souvenir héroïque. En dernière analyse, le martyre, en attribuant à l'idéologie une place supérieure à celle de la survie malgrès tout, déclare la priorité de la culture et de la communauté par rapport à la nature et à l'existence biologique.

Les récits juifs de la croisade témoignent que dans la culture juive d'Ashkenaz les pratiques de mémorisation et de commémoration sont narratives et rituelles; elles apportent la nouveauté de l'évocation des noms des héros contemporains et elles puisent dans un arsenal textuel et liturgique.

Venons-en maintenant aux récits de voyage. Le voyageur n'a pas forcement l'intention d'écrire I'histoire des lieux visités. Cependant, la description des villes, des monuments, des tombes, ou les témoignages permettent l'expression d'une idée sur le passé et sur l'histoire. Le récit de voyage participe de la construction d'une mémoire en reprenant des histoires anciennes pour les enrichir ou pour les transformer, mais il s'agit d'une mémoire qui ne concerne pas la curiosité pour le passé, une mémoire qui serait plûtot instrument de l'enseignement théologique et moral.

Les récits de voyage de Benjamin et de Petachia s'inscrivent dans le jeu entre l'histoire et la mémoire. La narration matérialise le souvenir en tant que pratique sociale. Le récit uniformise des fait ou des situations du passé en les transformant en "lieux" de mémoire pour établir une continuité entre eux. Le récit du voyage ressemble ainsi à un rituel de commémoration. Là encore, les voyageurs et les destinataires du récit rencontrent des symboles qui appartiennent au temps biblique pour s'unir dans une "communauté imaginaire".

Benjamin visite des villes, pas de "pays." C'est un familier de la culture urbaine; il signale toujours la situation démographique des communautés juives, leurs activités économiques, leurs institutions et leurs rapports avec les non juifs. II nomme les grands rabbins, les chefs de communauté. Sur les monuments il cherche toujours à identifier des traces d'une histoire juive et s'il n'en trouve pas il les invente. II ne s'interesse à l'histoire des pays visités que dans la mesure où cela lui permet d'y reconnaitre un passé juif, souvent lui aussi inventé. Ses observations perspicaces n'ont pas de perspective temporelle: il reconnait que la situation des juifs selon les lieux n'est pas la même mais il ne l'attribue pas à des histoires différentes. La mémoire dont il est question dans son récit ne dépend pas des pays, des institutions, des histoires locales: elle a une fonction utilitaire dans ce sens qu'elle permet aux communautés juives de se reconnaitre mutuellement en dépit de leur diversité.

En revanche, Petachia visite des "pays", il ignore les villes. Les lieux où les souvenirs se cristallisent sont pour ce voyageur les monuments, une tombe ou une synagogue; il y reconnait le sens d'une continuité interrompue par la Diaspora. L'histoire des peuples visités, à travers leurs propres monuments laisse Petachia indifférent. Selon J. Prawer, ${ }^{13}$ en énumerant les tombes 
des ancetres en Palestine les juifs révendiquent le pays; cette interprétation me parait anachronique; je dirais plutôt que les voyageurs révendiquent l'histoire et pas la géographie.

Le mouvement de pélèrinage aux lieux saints fait découvrir des tombes des ancêtres et inventer des traditions. Le parcours de Petachia s'inscrit comme un acte de pélèrinage.$^{14}$ Le voyageur rencontre son Dieu aux monuments de pays différents mais son récit les assemble en mémoire commune. De ce point de vue les pays étrangers deviennent familiers ou même homogènes. Petachia voyage dans des pays lointains mais il visite toujours son propre épicentre culturel. Lorsque les miracles manifestent la sainteté des lieux, l'imaginaire intervient entre I'histoire biblique et le présent du voyageur afin de renforcer sa mémoire. Le miracle empêche le temps d'effacer les traces.

Dans les deux récits, l'écriture sert d'intermédiaire entre la topographie biblique et la mémoire pour répondre au commandement biblique: zachor, souviens-toi. Le narrateur s'approprie symboliquement l'espace: le texte biblique s'inscrit dans la réalité du temps du voyageur. Benjamin est plutôt un observateur de la société, Petachia un interprète des signes. Le récit de ce dernier est plutôt motivé par la présence des monuments où se manifestent des signes surnaturels, en effacant la distance avec le passé biblique sans avoir à faire recours à la linéarité du récit historique. Les "sources" mobilisées par les deux récits diffèrent: les communautés pour l'un, les tombes et les miracles pour l'autre. Leurs différentes manières de voir-qui dépendent de leurs expériences sociales et culturelles à Sépharade ou en Ashkenaz-intégrent un passé lointain dans une tradition qu'ils sont en train de créer.

Le récit de voyage est aussi une pratique de commémoration. ënumération des communautés, noms des grands rabbins, tombes et monuments deviennent autant des "lieux de mémoire" qui trament l'identité collective.

\section{ii. Penser l'existence juive}

Dans la pensée rabbinique, les catastrophes s'inscrivent dans une continuité inélluctable. En mettant en valeur les malheurs qui coïncidèrent avec la date de la destruction du Temple, le 9 du mois de Av, on signale le besoin de désigner le temps de la première rupture dans le contrat qui unit à Dieu, une rupture qui annonce toutes celles qui vont suivre. Intégrer la catastrophe dans un continuum signifie aussi faire preuve d'un acte de foi collective.

Une nouvauté frappante, importante pour l'image de soi collective, caractèrise les récits juifs de la croisade. En 1096, la première réaction des juifs aux persécutions a été une intense surprise. Mais la surprise ne doit pas cependant ébranler la certitude des interprétations offertes par la foi. Une première réponse reprend l'antienne de la culpabilité humaine. II est fréquent à la fin du Xle siècle-aussi bien chez les juifs que chez les chrétiens - de considérer la catastrophe comme une punition de Dieu pour les péchés commis par les hommes. Dans les paroles que le narrateur met dans la bouche des croisés nous discernons l'argument de la polémique contre les juifs: puisque les juifs ont abandonnés Jésus, la grâce divine les a abandonné aussi; le verus Israel est 
représenté par les chrétiens. Le récit se transporte sur le terrain de la polémique théologique et une technique rhétorique permet d'écarter l'idée de culpabilité pour faire prévaloir l'interprétation de l'épreuve, et affirmer une attitude de confiance en soi et de fierté. La catastrophe est donc œuvre de Dieu "pour éprouver ceux qui ont peur de Lui”. Précisement, parce que les juifs sont un peuple élu, ils ont été choisis pour offrir un sacrifice, churban. Des thèmes bibliques sont appelés en renfort de cette interprétation puisque, dans la Bible déjà, les événements comtemporains sont toujours présentés comme une réactivation de faits antérieurs. Dans l'interprétation qui domine alors, la détresse des martyrs est liée à l' épreuve de Job. Finalement, les persécutions des croisés ne constituent pas une punition divine. Ceci n'est pas permis par la représentation positive que les juifs se font d'eux-mêmes, par la confiance en leur propre foi. Leur piété éloigne toute idée de culpabilité. 'A ce moment précis de la première croisade, la catastrophe ne signifie pas la punition d'une faute, elle s'identifie à une épreuve. II faut souligner cet aspect car il représente une innovation importante, une rupture, par rapport à la tradition narrative de la catastrophe aussi bien dans la Bible (Deutéronome et Lamentations), que dans la littérature rabbinique, le Midrach, où règnent les concepts de péché et de juste punition divine. Cette rupture par rapport à la tradition apporte un élement nouveau à l'image de soi. ${ }^{15}$

Cependant, la mort héroïque n'empêche ni la souffrance, ni le chagrin. Les lamentations rétablissent les rapports face à l'expérience de l'isolement et de la séparation. Avec les pleurs la douleur est vécue comme exposition au destin extérieur. Dans le récit les pleurs parlent de l'authenticité, de la vérité de cette douleur. ${ }^{16}$

Mais les lamentations sont surtout adressées à Dieu comme une plainte: "Oh étoiles pourquoi n'avez-vous pas retenu votre lumière? "Pourquoi les cieux ne se sont-ils pas obscurcis, pourquoi les étoiles n'ont-elles-pas éteind leur lumière, pourquoi le soleil et la lune ne se sont-ils pas obscurcis? La plainte éloigne encore une fois tout soupçon de culpabilité: "et toutes les richesses ne nous ont pas été utiles, ni le jeûne, ni la douleur, ni les lamentations ou la charité et personne n'a violé la loi - ni maître, ni prince - et même la sainte Torah n'a pas protégé les savants." Cette manière d'argumenter avec Dieu, cette colère qui transparaît dans les textes, est une colère rituelle qui donne au texte une allure profane, c'est une attitude déraisonnée. Par une technique d'inversion symbolique Dieu prend le rôle de celui qui viole l'alliance. Mais les paroles qui accompagnent les pleurs viennent surtout de la Bible: les Psaumes (94,1-2, 44, 23, 79, 10 et 12, 69, 25), les Lamentations (2, 20-21), Isaïe (13:5, 42:13), etc. Parler du désespoir donne un faible espoir pour soi-même et pour les autres. Les narrateurs juifs donnent du sens à la catastrophe et à la douleur qu'elle provoque en établissant une relation de continuité avec le passé. Une tension se crée ainsi entre un mythe d'éternel retour et une perspective linéaire. Là ils puisent l'espoir qu'un jour Dieu punira les ennemis.

Le récit du martyre et l'exaltation de la mort héroïque apportent à l'image de soi à transmettre une allure nouvelle, celle de la fierté. 
Par la diversité des communautés juives visitées, le voyage se prête aussi à une reflexion sur le sens de l'existence juive et permet de parler explicitement des valeurs de la vie juive.

Benjamin "voit" surtout des juifs. J'ai déjà mentionné plus haut le système narratif de Benjamin: la plupart du temps il note approximativement la population, il mentionne toujours les chefs de la communauté, il donne une liste des rabbins et des renseignements sur la situation économique des juifs. II note toujours la présence des institutions pour l'étude du Talmud. Ses références à la prospérité materielle sont mises en rapport soit avec les capacités de ces institutions soit avec les actes de bienfaisance qui assurent la cohésion de la communauté.

En bref, Benjamin reconnait trois valeurs aux communautés, aux Kehillot de la Diaspora occidentale: Essor culturel, puissance économique et charité. II n'est jamais question de confrontation ou de répression due à des raisons strictement religieuses. En d'autres termes, le récit de Benjamin revèle la conviction que la différence religieuse ne constitue pas une barrière infranchissable lorsque la situation économique et les bons rapports avec le pouvoir politique assurent l'essor de la vie communautaire. Son expérience sépharade a sans aucun doute influencé cette vision du monde.

Sa description du monde byzantin suit le même modèle narratif. Cependant, ici les relations avec le pouvoir politique ne sont pas harmonieuses. Benjamin évoque, sans commentaire, le "régime de répression" pour les juifs de Salonique et le manque d'autonomie qui équivaut à la répression dans le cas des juifs de Constantinople.

En Palestine, où monuments et références bibliques abondent, Benjamin a rencontré très peu des juifs. II n'existe pas de villes ou des communautés telles qu'on les connait en Occident.

Babylone est un lieu important pour les deux voyageurs. Dans I'Islam les juifs possèdent un centre politique et culturel, celui de Babylone, tandis qu'en Occident l'absence d'une entité politique forte a favorisé l'existence des communautés autonomes. Les juifs de Bagdad représentent une sorte de version idéale de la vie juive en Diaspora. Éssor culturel, relations pacifiques et égalitaires avec les Musulmans. La frontière entre juifs et musulmans est parfois invisible.

En dépit du fait que le judaïsme traditionnel n'a jamais renoncé à la prétention de constituer une civilisation d'importance œucuménique, en réalité les juifs se sont attachés à leurs cadres institutionnels propres, aux études, à l'observation rituelle de la religion, à la prière et à l'organisation communautaire; ils avaient choisi à ne pas participer dans l'histoire politique de leur temps. La recherche de l'imporrtance œucuménique de la religion juive et la rédemption politique étaient projétées dans un avenir messianique lointain.

De manière générale, les récits juifs de voyage obéïssent à ces principes, mais leur caractère particulier ouvre aussi d'autres chemins. Je pense que le récit de Benjamin initie une vision 
positive de la Diaspora tout à fait novatrice. Le message d'espoir et de consolation ne vient pas de l'attente d'une intervention divine ou de la rédemption messsianique; il s'agit d'une consolation qui résulte d'une perspective plus large de la vie juive. ${ }^{17}$ Benjamin rapporte la diversité du monde juif. Ses juifs sont aussi bien des marchants que des teinturiers, des étudiants, des hérétiques à Chypre ou des Noirs en Inde etc. II note les différences dans l'organisation des communautés, les caractéristiques locales. II y a lieu pour une Diaspora idéale où les juifs ne sont pas persécutés, où leur identité est assurée. II est séduit par ses correligionaires lorsque ceux-ci sont indépendants, lorsqu' ils possèdent un pouvoir matériel et intellectuel, c'est à dire lorsqu'ils ont les moyens de mettre en œuvre les valeurs mêmes de la vie juive.

Dans le récit de Petachia seul le judaïsme de Babylone et ses conditions de vie juive importent; le voyageur ne montre pas un intéret equivalent pour les autres communautés. Son regard évite la réalité du présent; à travers la mémoire et l'imaginaire il se réfugie dans le passé ou l'avenir.

Les différences dans le récit des voyageurs reflètent les différences des mondes d'où ils viennent. Cependant un fil conducteur commun traverse leurs visions du monde. A la question qu'est ce qu'il peut bien faire la différence entre les lieux qu'ils visitent ils donnent la même réponse: les juifs qui s'y trouvent. La situation des juifs est de manière explicite ou implicite le seul critère, le point de vue qui leur permet de contempler le monde. Ils s'approprient le monde en l'amenant à un cadre familier et utile d'où partent leurs représentations. Des représetations aux termes de pouvoir. Ni pour Benjamin ni pour Petachia la Terre d' Israël n'est le centre de leur reflexion sur la condition juive, de ce que nous appelerions judaïté. Les catégories à travers lequelles ils perçoivent la judaïté sont la piété, l'essor intellectuel et l' essor économique qui assure les deux premiers.

L'exil, la galut, n'est pas une réalité extérieure aux textes. D'ailleurs, la représentation de la Diaspora n'est que l'effet symbolique d'un récit qu'elle a elle-même crée, en accord avec les mondes d'où partent Benjamin et Petachia. La Diaspora n'a pas des connotations uniquement négatives. Et là réside, je crois, la particularité de ces récits juifs de voyage par rapport aux autres types de récit ou par rapport aux récits de pélèrinage.

Je pense que les récits juifs de voyage du Xlle siècle renouvellent la représentation de l' exil comme Diaspora à plusieurs centres qui n' a comme référents essentiels ni la géographie, ni le passé en tant que tels mais une tradition en construction et des valeurs contemporaines: essor intellectuel et économique de la communauté, négociaton des rapports de pouvoir avec les autorités hétérodoxes.

iii. les "autres"

La persécution active une guerre d'insultes. N.Z. Davis ${ }^{18}$ qui a étudié les rites de violence a bien montré, à propos des guerres de religion, que la violence religieuse est extrême car elle est liée 
aux valeurs fondamentales d'une société, au sens que la communauté done à son identité. Les martyrs juifs considèrent leurs actes comme imitation ou accomplissement des vies exemplaires, comme réactivation d'une institution exemplaire, le Temple, qui se reconstruit symboliquement.

Dans certains cas, les victimes qui refusent le baptême, se font tuer par l'ennemi. D'autres choisissent de mourir avant de tomber dans les bras des croisés et rivalisent pour qui sera le premier à mourir pour la grâce de Dieu; ils se suicident ou s'entretuent et le narrateur exalte leur solidarité dans le sacrifice. Certains tuent leurs enfants pour qu'ils ne soient pas obligés de se convertir. Lorsque les juifs tuent leurs enfants avant de se suicider eux-mêmes, ils réactivent symboliquement le rituel du Temple (Exode 29, 18-21): le massacre dans les bras des chrétiens aurait souillé l'offrande du sacrifice, tandis que les parents avec leurs propres mains le sanctifient. Quelquefois la mort rituelle a lieu dans les eaux d'une rivière ou par le feu; la mort religieuse puise dans un arsenal de traditions purificatrices et punitives que détaille le récit. Les martyrs sont des hommes ou des femmes cités nommément, à chaque fois ils éprouvent la douleur individuellement. Le martyrologue s'attache à des cas précis, il ne correspond pas à un scénario connu d'avance; les femmes y jouent un rôle primordial et les conduites sacrificielles se présentent comme spontanées.

Les actes des martyrs témoignent d'une conception selon laquelle les différences doctrinales ou coutumières constituent un gouffre abyssal qui rend impossible le choix de la conversion. La réponse extrême correspond à la violence de l'attaque. Une nouvelle frontière rituelle se lève pour séparer juifs et chrétiens. S'agit-il d'une sorte de réponse à l'argument qui fait de la mort de Jésus un sacrifice? Rappellons que du côté chrétien, le thème du martyre, qui fait partie aussi bien de la piété savante que de la tradition populaire, a probablement contribué à la préparation de la croisade, et à la diffusion de ses idéaux chez les contemporains. Dans les mentalités le martyre était la légitimation suprême de l' ethos chrétien: être chrétien signifiait aussi souffrir. Le martyre juif était-il aussi une réponse à la conception chrétienne du martyre?

Insultes et paroles méprisantes manifestent la haine et prolongent la polémique entre juifs et chrétiens. La violence verbale consiste à défendre la doctrine; les adversaires sont appellés blasphémateurs ou menteurs et la peur de la souillure est répandue. Des termes dépréciatifs reviennent pour peindre l'ennemi; le baptême équivaut à une "souillure dans des eaux impies" et l' église devient "maison d'idôlatrie." Les insultes purifient la communauté, humilient l'ennemi, limitent, dans l'imagination du moins, le mal qu'il provoque. Avec les malédictions - dans un vocabulaire qui vient du Lévitique et du Deutéronome - l'horreur est projetée sur le persécuteur: on exile l'ennemi en dehors du monde naturel et là-bas on peut se venger de lui. ${ }^{19}$

Contrairement à ce que plusieurs historiens ont cru, les insultes ne sont pas liées de manière simple aux extrémités qu'entraine le martyre, elles n'expriment pas simplement la haine pour une religion qui veut s'imposer de force. Ces expressions remplissent une fonction précise dans la détermination de l'attitude des juifs dans le monde chrétien. A. Sapir Abulafia a montré que la 
source de ces insultes est une version juive des Évangiles: "I'Histoire de Jésus" (Toledot Yeshu), écrite entre le IVe et le Ve siècle. ${ }^{20}$ Les juifs étaient familiarisés avec ce texte et ils s'en servaient dans un effort conscient pour repousser l'activité apostolique et défendre la cohésion de leur communauté. Les thèmes principaux de cette version sont la contestation de la Vierge, de la Trinité et de la divinité de Jésus. La violence verbale fait partie de la mort rituelle: on humilie l'ennemi en insultant son Dieu.

Des gestes de répulsion accompagnent la violence verbale. Les synagogues et les objets de culte deviennent la cible des croisés qui foulent aux pieds et déchirent les rouleaux de la Torah. II ne s'agit pas d'une violence aveugle; les croisés prouvent symboliquement que les objets de culte ne possèdent pas de pouvoir magique. Le déchirement des rouleaux de la Torah, qui est répété plusieurs fois dans nos récits, est une attaque matérielle et symbolique du judaïsme. De leur côté les juifs déchirent leurs vêtements en signe de deuil, ils soulèvent la Torah, l'embrassent et se demandent comment elle a pu tomber entre les mains des "incirconscrits" (Arelhim), c'est-à-dire des impurs. Les juifs appellent les croisés Tohim, "errants", ceux qui errent mais aussi ceux qui sont dans l'erreur, ils les qualifient d'ennemis de Dieu qui "s'ornent d'un symbole blasphématoire, une ligne verticale, sur une ligne horizontale...". Dans le cadre de la persécution, la disputatio, l'échange des arguments théologiques qui défendent la vérité religieuse se transforme en insultes et menaces.

La persécution des juifs ne s'est pas déroulée uniquement dans le cadre d'une guerre qui avait des motifs idéologiques, eschatologiques ou des motifs de conversion. C'était aussi un combat rituel, une guerre des insultes et des récits. La confrontation en temps de persécution est ritualisée, pas aveugle; la violence puise dans un arsenal textuel qui procure l' idéologie et les mots qui sont nécessaires à cette guerre de religion.

Revenons en aux récits de voyage. Le problème du narrateur-voyageur est toujours un problème de "traduction": comment inscrire l' "autre" de manière convaincante dans l'univers mental des destinataires du récit? Altérité et identité se trouvent alors piégées dans les tropes narratives qui voudraient prouver leur substance autonome. ${ }^{21}$

La littérature rabbinique offre un code de lecture du monde non juif. Rome est identifié à l'Edom biblique, les peuples arabes ou I'Islam sont qualifiés du nom Ismaël. Les partisans d'autres religions sont appelés "idôlatres"; le judaïsme condamne plutôt des actions que des idées. Ce code, repris dans les récits de voyage fait aussi partie d'une guerre des récits entre les religions qui a certainement influencé les attitudes collectives. Notons que le récit des voyageurs correspond à une logique que nous rencontrons aussi dans les récits chrétiens. Le but serait de faire la "preuve" d'une supériorité culturelle qui s'appuie sur des "catégories naturelles" et qui établit symboliquemet des rapports de force. Benjamin par exemple reprend à propos des Byzantins tous les stéréotypes que les textes occidentaux leur attribuent: richesses et luxe qui sont accompagnés de mollesse. Benjamin est le premier Européen à parler des Druzes et Petachia devient "ethnologue" pour décrire la façon dont les femmes pleurent en Crimée. Sa 
description des habitants d'Ucranie rappelle de manière impressionnante les descriptions des Mongoles dans les récits chrétiens du XIle et du XIIle siècle. Pour se représenter les autres on a toujours recours à des stéréotypes et à des catégories historiques et culturelles fondamentales, telles que la nourriture.

Le fantastique rend lui aussi service aux efforts de construire une supériorité: par leur puissance magique, miraculeuse, des tombes peuvent revèler la "vérité" et même punir les "infidèles" qui mettent en doute cette qualité.

La loi juive ne nie pas le salut aux non juifs, elle n'aspire pas non plus à leur conversion. Cependant, le thème de la conversion au judaisme me paraît important en ce qui revèle l'intentionnalité ou la fluidité de l'identité, son articulation avec les structures du mouvement historique. La religion juive semble ne pas rechercher la conversion d'une manière apostolique bien qu'il ya eu des périodes où des groupes importants se sont convertis au judaïsme. La loi rabbinique accepte les convertis et Maïmonide a une position favorable à leur égard. Au Moyen Age, le peuple par excellence sur lequel le rêve de conversion est projété est celui des Khazares, un groupe ethnique d'origine turque. Ils se sont probablement convertis au judaïsme en 740. Au Xe siècle il y a eu un échange des lettres entre le roi des Khazars Joseph et Hisdai Ibn Sharput de Cordova, diplomate au service du chaliphe. Sur cette correspondance s'appuie l'œuvre de Yehuda Halevi, "Kuzari", dialogue philosophique dramatisé qui aurait eu lieu devant le roi des Khazares, qui après avoir entendu les représentants des différentes religions décide de se convertir et il est suivi par son peuple. Les juifs étaient peut-être flattés à l'idée d'un royaume juif indépendant ou même ils attendaient des avantages pour leur commerce. Mais je pense aussi qu'on peut discerner une attitude très semblable à celle des chrétiens à l'égard des Mongoles, qui revèle le besoin d'imaginer un allié puissant qui en même temps reconnait la supériorité de leur religion. ${ }^{22}$

Il faudrait cependant insister sur un point. Les voyageurs juifs cherchent des juifs. Dans les villes d'Occident, Benjamin ne parle jamais des chrétiens en tant que tels et en Syrie ou au Liban il n'est pas préoccupé par les musulmans. Le chaliphe de Bagdad est la seule autorité non juive qui attire le respect et l'admiration de Benjamin, mais son importance est aussi due au fait que lui aussi reconnait l'Exilarche. De même, selon Petachia Bagdad est le seul lieu où un certain sycrétisme est favorisé.

En ce qui concerne les pratiques de représentation des "autres" les récits juifs de voyage présentent des similitudes avec les récits chrétiens, mais on peut discerner une différence selon l'importance qui leur est attribuée: décidement, c'est surtout aux juifs que les voyageurs juifs s'interressent!

En guise de conclusion:

Les éléments fragmentaires qui se dégagent des différentes sources dont je disposais présentent, à mon avis, un avantage: ils nous invitent à nous éloigner de l'illusion d'une culture homogène (la culture juive du Xlle siècle, dans ce cas), mais en même temps ils suggèrent des "liens souterrains" et je veux dire par là des pratiques de représentation ou des perceptions qui 
sont plus ou moins stables. Dans ce sens, nous pouvons nous interroger sur la logique qui chaque fois dicte les choix: l'observation et l'invention de la tradition mais aussi les "emprunts" qui traversent les frontières entre les communautés et les cultures; "no culture is an island," et même si les hommes et les femmes concernés pensent le contraire, des idées et des formes de pensée passent sans cesse les frontières.

Dans cette perspective j' essaierai de tirer quelques conclusions ou plutôt formuler quelques hypothèses en partant des récits juifs de persécution ou de voyage.

1. Les pratiques de mémorisation et celles de commémoration sont un élement fondamental dans le processus de construction de l'identité juive en temps de guerre comme en temps de paix. II y a là une pratique narrative et sociale qui prend sa source dans le récit biblique. Cependant, les noms des martyrs, ceux des grands rabbins ou bien les tombes et les monuments juifs deviennent des nouveaux "lieux de mémoire" et de cette manière les récits du XIle siècle renouvellent une tradition juive qui par ailleurs n'est pas pour autant homogène dans ses manières narratives.

2. Les identités collectives sont toujours en construction, relatives et imparfaites, c'est à dire hétérogènes; elles se forment dans le cadre des représentations, jamais en déhors d'elles. Les pratiques de représentation d'un soi collectif, la reflexion sur le sens de l'existence juive, dépend des rapports de force avec les communautés voisines. Les récits de persécution apportent un sens nouveau de fierté collective, les récits de voyage apportent une représentation nouvelle de la Diaspora qui n'est plus forcément dépréciative, qui revendique une sociabilité ouverte.

3. Les pratiques de représentation des "autres", sont instrument de polémique en temps de persécution ou simplement hierarchisées dans le cadre d'un voyage. II faut cependant noter que, même si les récits juifs de voyage présentent des traits communs avec les récits chrétiens, les voyageurs juifs s' interessent avant tout aux communautés juives, à la mémoire juive.

Les problèmes que nous nous sommes posés sont aussi liés au sujet de "l'individu au Xlle siècle," à cette "émergence du moi" dont on a souvent parlé. C. Bynum ${ }^{23}$ a montré, à propos des chrétiens, qu'il n'est pas question d'une "autonomie de l'individu" mais d'une nouvelle possibilité de choisir entre les identités des groupes différents. Je crois que les récits juifs nous invitent à réflechir sur cette perspective: II apparait que si augourd'hui nous voulons suivre les traces d'une "identité juive au Xlle siècle" nous le faisons sur les limites d'une tradition textuelle qui est toujours en mouvement: tantôt elle s'inspire des textes, tantôt elle obéit à une stratégie de polémique ou de dialogue, tantôt elle apparait comme le résultat des emprunts explicites ou bien souterrains.

\footnotetext{
${ }^{1}$ Notre communication s'appuie essentielement sur nos deux études précédentes: "Fierté, désespoir et mémoire: Les récits juifs de la première croisade", Médiévales, 35 (automne 1998), pp.125-140 et Voyageurs juifs au Moyen Age, à paraitre en grec, Athènes: Nepheli, 1999.
} 
${ }^{2}$ Nous possédons par ordre chronologique les éditions suivantes: A Neubaer R et $\mathrm{H}$. Stern (éds), Hebräishe Berichte über die Judenverfolgungen der Kreuzzüge. Berlin, 1892; A.M. Habermann (éd.), Sefer Gezerot Ashkenaz ve-Zarfat. Jérusalem, 1945; S. Eidelberg (éd.), The Jews and the Crusaders. The Hebrew Chronicles of the First and Second Crusades. Wisconsin, 1977; R. Chazan, traduction en Appendice dans le: European Jewry and the First Crusade. Berkeley, Los Angeles, 1987, pp. 223-297. Nous avons consulté les éditions de Eidelberg et de Chazan et le texte hebraïque établit par l'édition de Neubauer-Stern.

${ }^{3}$ Nous possédons les éditions suivantes: A. Asher, The itinerary of Benjamin of Tudela. vol. 2, LondonBerlin,1840-1842; Grünhut-M. Adler, Die Reisebeshreibungen des Rabbi Benjamin von Tudela. vol. 2, Jerusalem-Franncfurt, 1903-1904; Marcus Nathan Adler, The itinerary of Benjamin of Tudela. Oxford, 1907. (traduction reprise par M.A. Signer (ed.), The Itinerary of Benjamin of Tudela. Travels in the Middle Ages. Malibu California, 1987); S. E. Carmoly, Itinéraires de la terre sainte des XIIle et XIVe-XVe-XVIe-XVIIe siècles. Paris, sans date (traduits de l'hébreu).

${ }^{4}$ Nous possédons les éditions suivantes: L. Grünhut, Die Rundreise der R. Petachjah aus Regensburg, Frangfurt-Jerusalem, 1905 (en hebrew); A. Benisch (éd.), The Travels of Rabbi Petachia of Ratisbon. London, 1856; Elkhan Récement les deux textes ont été édité en français par H. Harboun, Les voyageurs juifs au Moyen Age. XII' siècle. Aix-en-Provence, 1986 et par J. Shatzmiller, dans Croisades et pèlerinages, Récits chroniques et voyages en terre sainte XIle-XVle siècle. dir. D. Régnier-Bohler, Paris, 1997, pp. 12811343.

${ }^{5}$ K. R. Stow, Alienated Minority. The Jews of Medieval Latin Europe. London, 1992, pp. 102-120.

${ }^{6} \mathrm{H}$. Benveniste, "Esquisse d'une histoire de la responsabilité dans les récits juifs de persécution", Historein, 1 (1999), pp. 113-124.

${ }^{7}$ I. G. Marcus, "From Politics to Martyrdom. Shifting Paradigms in the Hebrew Narratives of the 1096 Crusade Riots", Prooftexts, 2 (1982), pp. 40-52.

${ }^{8}$ D. G. Roskies, Against the Apocalypse. Responses to Catastrophe in Modern Jewish Culture. Cambridge Mass., 1984, pp. 47-48.

${ }^{9}$ R. Chazan, "Representation of Events in the Middle Ages", History and Theory, XXVII (1988), p. 41.

${ }^{10}$ I.G. Marcus, Rituals of Childwood. Jewish Acculturation in Medieval Europe. New Haven, London, 1996, pp. 1-17.

${ }^{11}$ I.G. Marcus, "Une communauté pieuse et le doute: mourir pour la Sanctification du Nom en Achkenaz et I'histoire de rabbi Amnon de Mayence", Annales ESC, 49:5 (1994), pp. 1031-1047.

${ }^{12}$ J.Cl. Schmitt, "Le suicide au oyen Age", Annales E.S.C., 31 (1976), pp. 3-28.

${ }^{13} \mathrm{~J}$. Prawer, The History of the Jews in the latin Kingdom of Jerusalem. Oxford, 1988.

${ }^{14}$ Y. Levanon, The Jewish Travellers in the Twelfth Century. University Press of America, 1980.

${ }^{15} \mathrm{H}$. Benveniste, "Esquisse d'une histoire de la responsabilité dans les récits jiufs de persécution", op.cit. ${ }^{16}$ ibid.

${ }^{17}$ M.A. Signer, "Introduction", The Itinerary of Benjamin of Tudela. Travels in the Middle Ages. Malibu, California, 1987.

${ }^{18}$ N. Z. Davis, "Les rites de violence”, Les cultures du Peuple. Rituels, savoirs et résistances au XVIe siècle. Paris, 1979, pp. 251-307. 
${ }^{19} \mathrm{H}$. Benveniste, "Fierté, désespoir et mémoire: Les récits juifs de la première croisade", op.cit.

${ }^{20}$ A. Sapir Abulafia, "Invectives against Christianity in the Hebrew Chronicles of the First Crusade" dans P.W. Edbury (ed.), Crusade and Settlement. Cardiff, 1985, pp. 66-72.

${ }^{21}$ Voir aussi H. Benveniste, “Joinville te les “autres": les procédés de représenytions dans l'Histoire de saint Louis”, Le Moyen Age, Cll:1 (1996), pp. 27-55.

${ }^{22} \mathrm{H}$. Benveniste, Voyageurs juifs au Moyen Age. op.cit.

${ }^{23}$ C.W. Bynum, "Did the 12th Century Discover the Individual", Journal of Ecclesiastical History, $31: 1$ (1980) pp. 1-12. 